TRANSACTIONS OF THE

AMERICAN MATHEMATICAL SOCIETY

Volume 357, Number 11, Pages 4647-4659

S 0002-9947(04)03652-9

Article electronically published on December 28, 2004

\title{
UNIPOTENT FLAT BUNDLES AND HIGGS BUNDLES OVER COMPACT KÄHLER MANIFOLDS
}

\author{
SILKE LEKAUS
}

\begin{abstract}
We characterize those unipotent representations of the fundamental group $\pi_{1}(X, x)$ of a compact Kähler manifold $X$, which correspond to a Higgs bundle whose underlying Higgs field is equal to zero.

The characterization is parallel to the one that $\mathrm{R}$. Hain gave of those unipotent representations of $\pi_{1}(X, x)$ that can be realized as the monodromy of a flat connection on the holomorphically trivial vector bundle.

We see that Hain's result and ours follow from a careful study of Simpson's correspondence between Higgs bundles and local systems.
\end{abstract}

\section{INTRODUCTION}

For a smooth, complex projective variety $X, \mathrm{R}$. Hain investigated in 2] when a unipotent group homomorphism $\rho: \pi_{1}(X, x) \rightarrow \mathrm{Gl}(n, \mathbb{C}), x \in X$, can be realized as the monodromy representation of a flat connection on the holomorphically trivial bundle.

Since $\rho$ is assumed to be unipotent, its $\mathbb{C}$-linear extension to $\mathbb{C} \pi_{1}(X, x)$ equivalently factors through a power $J^{s+1}$ of the augmentation ideal (see Section 3). The quotient $\mathbb{C} \pi_{1}(X, x) / J^{s+1}$ carries a mixed Hodge structure as defined by Morgan ([5]) and Hain ([3]).

Hain proved that a unipotent $\rho$ can be realized as the monodromy of a flat connection on the holomorphically trivial bundle, if and only if its $\mathbb{C}$-linear extension factors through the ideal

$I:=J \cap \mathrm{F}^{0}\left(\frac{\mathbb{C} \pi_{1}(X, x)}{J^{s+1}}\right)+J^{2} \cap \mathrm{F}^{-1}\left(\frac{\mathbb{C} \pi_{1}(X, x)}{J^{s+1}}\right)+\cdots+J^{s} \cap \mathrm{F}^{-(s-1)}\left(\frac{\mathbb{C} \pi_{1}(X, x)}{J^{s+1}}\right)$,

where $\mathrm{F}^{\bullet}$ denotes the Hodge filtration. This naturally leads to considering the ideal

$\bar{I}=J \cap \overline{\mathrm{F}}^{0}\left(\frac{\mathbb{C} \pi_{1}(X, x)}{J^{s+1}}\right)+J^{2} \cap \overline{\mathrm{F}}^{-1}\left(\frac{\mathbb{C} \pi_{1}(X, x)}{J^{s+1}}\right)+\cdots+J^{s} \cap \overline{\mathrm{F}}^{-(s-1)}\left(\frac{\mathbb{C} \pi_{1}(X, x)}{J^{s+1}}\right)$.

We ask the question if also the property of a unipotent group homomorphism to factor through the ideal $\bar{I}$ can be expressed in a corresponding category of bundles. In this article we show that this is possible, namely in the category of Higgs bundles, when $X$ is a compact, complex Kähler manifold.

A Higgs bundle over a compact, complex Kähler manifold $X$ is defined to be a pair $(E, \theta)$, where $E$ is a holomorphic vector bundle over $X$ and $\theta: E \rightarrow E \otimes \Omega_{X}^{1}$ is a holomorphic map such that $\theta \wedge \theta=0$. Higgs bundles play an important role in non-abelian Hodge theory.

Received by the editors October 31, 2003 and, in revised form, January 24, 2004.

2000 Mathematics Subject Classification. Primary 14F05, 14C30, 32Q20.

(C)2004 American Mathematical Society 
In [7], C. Simpson proves that over a compact, complex Kähler manifold there is an equivalence of categories between the category of flat bundles and the category of Higgs bundles that are extensions of stable Higgs bundles with vanishing Chern classes.

We show that using Simpson's correspondence Hain's result translates into

Theorem 1.1 (Hain, 2]). Let $X$ be a compact, complex Kähler manifold and $(V, \nabla)$ a flat bundle whose $\mathbb{C}$-linear extension $\rho$ of the monodromy representation factors through $J^{s+1}$.

Then $\rho$ factors through $I$ if and only if the underlying holomorphic bundle of any representative of the isomorphism class of Higgs bundles corresponding to $(V, \nabla)$ is trivial.

The main result of this article is

Theorem 1.2. Let $X$ be a compact, complex Kähler manifold and $(V, \nabla)$ a flat bundle whose $\mathbb{C}$-linear extension $\rho$ of the monodromy representation factors through $J^{s+1}$.

Then $\rho$ factors through $\bar{I}$ if and only if any representative of the isomorphism class of Higgs bundles corresponding to $(V, \nabla)$ has Higgs field equal to zero.

In fact, Hain's theorem holds if $X$ is the complement of a normal crossing divisor. However, since we are using Simpson's correspondence, established essentially only for compact, complex Kähler manifolds, we are only considering this case.

Sections [2, 3] and 4 serve to provide the reader with background material about the correspondence proved by Simpson, unipotent representations and the mixed Hodge structure on $\mathbb{C} \pi_{1}(X, x) / J^{s}$ and to fix the notation used in later paragraphs. There are no new results in these sections and they can be skipped by the reader familiar with these topics.

In Section [5, we shortly describe the special case of bundles whose monodromy factors through $J^{2}$, which illustrates the method of the proofs in the general case, which is done in Section 6 .

\section{Correspondence Between flat Bundles and Higgs Bundles}

Let $X$ be a compact complex Kähler manifold. A Higgs bundle $(E, \theta)$ over $X$ as defined in the introduction has an equivalent $\mathcal{C}^{\infty}$-description (see [7]): It can be considered as a $\mathcal{C}^{\infty}$-bundle with a first order operator $D^{\prime \prime}=\bar{\partial}+\theta$, where $\bar{\partial}$ defines the holomorphic structure of $E$ by taking sections of $E$ to $(0,1)$-forms with coefficients in $E$ and by annihilating holomorphic sections. It holds that $D^{\prime \prime}$ is integrable, i.e., $\left(D^{\prime \prime}\right)^{2}=0$, because $\bar{\partial}^{2}=0, \theta \wedge \theta=0$, and $\bar{\partial}(\theta)=0$, as $\theta$ is holomorphic. Furthermore, it fulfills the Leibniz rule

$$
D^{\prime \prime}(f e)=\bar{\partial}(f) \wedge e+f D^{\prime \prime}(e)
$$

for all sections $f$ of $\mathcal{A}^{0}$ and all sections $e$ of $E$.

Conversely, let $D^{\prime \prime}=\bar{\partial}+\theta$ be an operator on a $\mathcal{C}^{\infty}$-vector bundle $E$, so that $\bar{\partial}$ takes sections of $E$ to $(0,1)$-forms with coefficients in $E$ and $\theta$ takes sections of $E$ to $(1,0)$-forms with coefficients in $E$. Such an operator defines a Higgs structure on $E$, if and only if it is integrable and fulfills the Leibniz rule. These conditions imply that $\bar{\partial}^{2}=0, \theta \wedge \theta=0$, and that $\theta$ is holomorphic.

The categories of flat bundles and Higgs bundles over $X$ form so-called differential graded categories, if we define an appropriate Hom-complex (see 7]) as follows. 
Example 2.1. (1) By $\mathcal{C}_{\mathrm{DR}}$ we denote the differential graded category whose objects are all flat bundles over $X$ and which has the Hom-complex

$$
\operatorname{Hom}^{\bullet}(U, V)=\left(\Gamma\left(X, \mathcal{A}^{\bullet}(\operatorname{Hom}(U, V))\right), D\right),
$$

where the composition of homomorphisms is given by the wedging of forms.

(2) By $\mathcal{C}_{\mathrm{DR}}^{s}$ we denote the full subcategory of $\mathcal{C}_{\mathrm{DR}}$ consisting of semisimple objects.

(3) By $\mathcal{C}_{\text {Dol }}$, we denote the differential graded category whose objects are those Higgs bundles on $X$ which are extensions of stable Higgs bundles with vanishing Chern classes. Its Hom-complex is

$$
\operatorname{Hom}^{\bullet}(U, V)=\left(\Gamma\left(X, \mathcal{A}^{\bullet}(\operatorname{Hom}(U, V))\right), D^{\prime \prime}\right),
$$

where the composition of morphisms is obtained by the wedging of forms.

(4) By $\mathcal{C}_{\text {Dol }}^{s}$ we denote the full subcategory consisting of semisimple objects, i.e., polystable Higgs bundles.

(5) By $\mathcal{C}_{\text {harm }}^{s}$ we denote the category of all harmonic bundles (i.e., those bundles which carry both a flat and a Higgs structure) with Hom-complex

$$
\operatorname{Hom}^{\bullet}(U, V)=\left(\operatorname{ker}\left(D^{\prime}\right) \subset \Gamma\left(X, \mathcal{A}^{\bullet}(\operatorname{Hom}(U, V))\right), D^{\prime \prime}\right),
$$

where $D$ is the flat operator and $D^{\prime \prime}=\bar{\partial}+\theta$ the Higgs operator on $\operatorname{Hom}(U, V)$, and $D^{\prime}:=D-D^{\prime \prime}$.

If $\mathcal{C}$ is a differential graded category with differential $d$ on the Hom-complex, we can construct its completion $\hat{\mathcal{C}}$ (see [1], 7]):

Let $\overline{\mathcal{C}}$ be the differential graded category, whose objects are pairs $(U, \eta)$ with an object $U$ of $\mathcal{C}$ and an endomorphism $\eta \in \operatorname{Hom}^{1}(U, U)$ of degree one, satisfying $d(\eta)+\eta^{2}=0$. If $(U, \eta)$ and $(V, \xi)$ are two objects of $\overline{\mathcal{C}}$, we define the differential graded algebra $\operatorname{Hom}((U, \eta),(V, \xi))$ to be $\operatorname{Hom}(U, V)$ with the same grading, the new differential $\hat{d}(f)=d(f)+\xi f-(-1)^{\operatorname{deg}(f)} f \eta$.

The category $\mathcal{C}$ can be embedded into $\overline{\mathcal{C}}$ by mapping an object $U$ to $(U, 0)$.

We define the completion $\hat{\mathcal{C}}$ of $\mathcal{C}$ to be the full subcategory of $\overline{\mathcal{C}}$, whose objects are successive extensions of objects of $\mathcal{C}$.

There is the following lemma:

Lemma 2.2 (Simpson, [7]). There are equivalences of differential graded categories

$$
\hat{\mathcal{C}}_{\mathrm{DR}}^{s} \cong \mathcal{C}_{\mathrm{DR}} \text { and } \hat{\mathcal{C}}_{\mathrm{Dol}}^{s} \cong \mathcal{C}_{\mathrm{Dol}} \text {. }
$$

Remark 2.3. In the following we will need a concrete description of the functors

$$
\hat{\mathcal{C}}_{\mathrm{DR}}^{s} \rightarrow \mathcal{C}_{\mathrm{DR}} \text { and } \hat{\mathcal{C}}_{\mathrm{Dol}}^{s} \rightarrow \mathcal{C}_{\mathrm{Dol}},
$$

which can be found in [7], Section 3:

Let a pair $((U, D), \eta)$ be an object of $\hat{\mathcal{C}}_{\mathrm{DR}}^{s}$, i.e., $(U, D)$ is a semisimple flat bundle and $\eta$ is 1 -form with values in $\operatorname{End}(U)$ satisfying $D(\eta)+\eta \wedge \eta=0$. The corresponding (in general not semisimple) object of $\mathcal{C}_{\mathrm{DR}}$ is $(U, D+\eta)$.

Analogously, an object $\left(\left(U, D^{\prime \prime}\right), \eta\right)$ of $\hat{\mathcal{C}}_{\text {Dol }}^{s}$ is mapped to $\left(U, D^{\prime \prime}+\eta\right)$.

If $(\mathcal{C}, d)$ is a differential graded category, we denote by $\mathrm{E}^{0}(\mathcal{C})$ the additive category whose objects are the same as those of $\mathcal{C}$, but whose morphisms from $U$ to $V$ are just $\operatorname{Ext}^{0}(U, V)=\operatorname{Ker} d: \operatorname{Hom}^{0}(U, V) \rightarrow \operatorname{Hom}^{1}(U, V)$. 
Lemma 2.4 (Simpson, [7]). There is an equivalence of categories between the category $\mathrm{E}^{0}\left(\mathcal{C}_{\mathrm{DR}}\right)$ of flat bundles and the category $\mathrm{E}^{0}\left(\mathcal{C}_{\mathrm{Dol}}\right)$ of Higgs bundles which are extensions of stable Higgs bundles with vanishing Chern classes. Furthermore, these are equivalent to the category $\mathrm{E}^{0}\left(\hat{\mathcal{C}}_{\text {harm }}^{s}\right)$.

Remark 2.5. The equivalence $\mathrm{E}^{0}\left(\mathcal{C}_{\mathrm{DR}}\right) \cong \mathrm{E}^{0}\left(\mathcal{C}_{\mathrm{Dol}}\right)$ can be described by using the equivalence to $\mathrm{E}^{0}\left(\hat{\mathcal{C}}_{\text {harm }}^{s}\right)$ (as suggested by Corlette, see [7]):

An object of $\mathrm{E}^{0}\left(\hat{\mathcal{C}}_{\text {harm }}^{s}\right)$ is a pair $(U, \eta)$ with a harmonic bundle $U=\left(U, D, D^{\prime}, D^{\prime \prime}\right)$ and $\eta \in \Gamma\left(X, \mathcal{A}^{1}(\operatorname{End}(U))\right.$ such that $D^{\prime}(\eta)=0$, and $D^{\prime \prime}(\eta)+\eta \wedge \eta=0$. A pair $(U, \eta)$ is mapped to the Higgs bundle $\left(U, D^{\prime \prime}+\eta\right)$ and to the flat bundle $(U, D+\eta)$. Since these functors are equivalences of categories, for any flat bundle and for any Higgs bundle, satisfying the conditions of the lemma, there is a unique pair $(U, \eta)$ which maps to it.

Note: Since a harmonic bundle is semisimple as a flat bundle (see [7, Theorem 1), a unipotent harmonic bundle $U$ is necessarily isomorphic to a direct sum of trivial bundles $\left(\mathcal{A}_{X}^{0}, d\right)^{\oplus r}$. Thus there exists a global flat frame for $U$, and with respect to this frame $D=d, D^{\prime}=\partial$, and $D^{\prime \prime}=\bar{\partial}$ are the usual differential operators operating coordinatewise.

\section{UNIPOTENT REPRESENTATIONS AND UNIPOTENT FLAT BUNDLES}

Let $G$ be a group. The homomorphism of $G$ to the trivial group induces the algebra homomorphism

$$
\begin{aligned}
\epsilon: \mathbb{C} G & \rightarrow \mathbb{C}, \\
\sum \alpha_{g} g & \rightarrow \sum \alpha_{g} .
\end{aligned}
$$

The kernel

$$
J:=\operatorname{Ker}(\epsilon)
$$

is called the augmentation ideal. It is spanned by $\{g-1 \mid g \in G\}$.

Let $V$ be a finite-dimensional $\mathbb{C}$-vector space and $\rho: G \rightarrow \operatorname{Aut}(V)$ a representation. It can be extended by linearity to an algebra homomorphism

$$
\bar{\rho}: \mathbb{C} G \rightarrow \operatorname{End}(V) .
$$

Proposition 3.1. The following are equivalent:

(1) $\rho$ is unipotent.

(2) $\rho$ induces a homomorphism

$$
\bar{\rho}: \mathbb{C} G / J^{s+1} \rightarrow \operatorname{End}(V)
$$

for some $0 \leq s \leq \operatorname{dim} V$.

Proof. This follows from Kolchin's theorem (see e.g. 6], Part I, Chapter V), saying that there is a basis for $V$, in which all elements of $\rho(G)$ are simultaneously represented by upper-triangular matrices with 1's on the diagonal.

In the following $X$ denotes again a compact complex Kähler manifold.

Definition 3.2. A flat bundle $(V, \nabla)$ over $X$ is called unipotent, if its monodromy representation $\rho$ is unipotent.

The unipotent flat bundles are exactly those, which are successive extensions of $\left(\mathcal{A}_{X}^{0}, d\right)$ by itself, as follows from the following proposition. 
Proposition 3.3. There is an equivalence of categories between the category of unipotent representations of $\pi_{1}(X, x)$ and the category of flat bundles, which are successive extensions of $\left(\mathcal{A}_{X}^{0}, d\right)$.

The correspondence is the usual Riemann-Hilbert correspondence.

Proof. This holds since the Riemann-Hilbert correspondence respects exact sequences.

\section{The REDUCED BAR CONSTRUCTION AND THE MiXed \\ Hodge STRUCTURe ON $\mathrm{H}^{0}\left(B_{s}\left(\mathcal{A}^{\bullet}\right)\right)$}

We explain the reduced bar construction following [4].

Let $A^{+}:=\bigoplus_{i=1}^{\infty} \Gamma\left(X, \mathcal{A}^{i}\right)$. For each $s \geq 0$ we define

$$
T^{-s, t}:=\left[\bigotimes^{s} A^{+}\right]^{t},
$$

where $t$ is the sum of the degrees of the elements in $A^{+}$. We denote an element $\omega_{1} \otimes \cdots \otimes \omega_{s}$ by $\left[\omega_{1}|\ldots| \omega_{s}\right]$ and give it the total degree $s-t$.

On every $T^{-s, t}$ we define two differentials, namely the combinatorial differential $d_{C}: T^{-s, t} \rightarrow T^{-s+1, t}$ given by

$$
d_{C}\left(\left[\omega_{1}|\ldots| \omega_{s}\right]\right):=\sum_{i=1}^{s-1}(-1)^{i+1}\left[J \omega_{1}|\ldots| J \omega_{i-1}\left|J \omega_{i} \wedge \omega_{i+1}\right| \omega_{i+2}|\ldots| \omega_{s}\right],
$$

and the internal differential $d_{I}: T^{-s, t} \rightarrow T^{-s, t+1}$ given by

$$
d_{I}\left(\left[\omega_{1}|\ldots| \omega_{s}\right]\right):=\sum_{i=1}^{s}(-1)^{i}\left[J \omega_{1}|\ldots| J \omega_{i-1}\left|d \omega_{i}\right| \omega_{i+1}|\ldots| \omega_{s}\right],
$$

where $J: A^{+} \rightarrow A^{+}$denotes the involution $J(\omega):=(-1)^{\operatorname{deg} \omega} \cdot \omega$.

It holds that

$$
d_{I}^{2}=d_{C}^{2}=0, d_{I} d_{C}+d_{C} d_{I}=0,
$$

such that $\left(T^{\bullet \bullet}, d_{I}, d_{C}\right)$ is a double complex.

We denote the total complex by $T\left(\mathcal{A}^{\bullet}\right)$.

We define $R$ to be the graded subspace of $T\left(\mathcal{A}^{\bullet}\right)$ spanned by the elements of the form

(a) $\left[d f\left|\omega_{1}\right| \ldots \mid \omega_{s}\right]+f(x)\left[\omega_{1}|\ldots| \omega_{s}\right]-\left[f \omega_{1}|\ldots| \omega_{s}\right]$,

(b) $\left[\omega_{1}|\ldots| \omega_{i-1}|d f| \omega_{i}|\ldots| \omega_{s}\right]+\left[\omega_{1}|\ldots| f \omega_{i-1}\left|\omega_{i}\right| \ldots \mid \omega_{s}\right]$

$-\left[\omega_{1}|\ldots| \omega_{i-1}\left|f \omega_{i}\right| \ldots \mid \omega_{s}\right]$

(c) $\left[\omega_{1}|\ldots| \omega_{s} \mid d f\right]+\left[\omega_{1}|\ldots| f \omega_{s}\right]-\left[\omega_{1}|\ldots| \omega_{s}\right] f(x)$.

The reduced bar construction $\bar{B}\left(\mathcal{A}^{\bullet}\right)$ is defined to be the quotient $T\left(\mathcal{A}^{\bullet}\right) / R$.

Defining

$$
\mathcal{T}^{-s}=\bigoplus_{u \leq s} T^{-u, v}
$$

for each $s \geq 0$, we obtain the filtration

$$
T\left(\mathcal{A}^{\bullet}\right) \supseteq \cdots \supseteq \mathcal{T}^{-2} \supseteq \mathcal{T}^{-1} \supseteq \mathcal{T}^{0}=\mathbb{C} \supseteq 0
$$

of $T\left(\mathcal{A}^{\bullet}\right)$, which induces the bar filtration

$$
\bar{B}\left(\mathcal{A}^{\bullet}\right) \supseteq \cdots \supseteq \bar{B}_{2} \supseteq \bar{B}_{1} \supseteq \bar{B}_{0}=\mathbb{C} \supseteq 0
$$

of $\bar{B}\left(\mathcal{A}^{\bullet}\right)$. 
We denote by $\mathrm{H}^{0}\left(B_{s}\left(\mathcal{A}^{\bullet}\right)\right):=\mathbb{C} \oplus \mathrm{H}^{0}\left(\bar{B}_{s}\left(\mathcal{A}^{\bullet}\right)\right)$ the set of classes of iterated integrals of at most length $s$ which are homotopy functionals, when restricted to loops at $x$.

An iterated integral is a sum

$$
c+\sum_{k=1}^{n_{1}} \int \omega_{k}^{(1)}+\sum_{k=1}^{n_{2}} \int \omega_{k 1}^{(2)} \omega_{k 2}^{(2)}+\cdots+\sum_{k=1}^{n_{s}} \int \omega_{k 1}^{(s)} \omega_{k 2}^{(s)} \cdots \omega_{k s}^{(s)}
$$

with $c \in \mathbb{C}$ and $\omega_{k i}^{(j)} \in \Gamma\left(X, \mathcal{A}_{X}^{1}\right)$.

It is homotopy invariant, when restricted to loops based at $x$, if and only if

$$
\left(d_{C}+d_{I}\right)\left(\sum_{k=1}^{n_{1}}\left[\omega_{k}^{(1)}\right]+\sum_{k=1}^{n_{2}}\left[\omega_{k 1}^{(2)} \mid \omega_{k 2}^{(2)}\right]+\cdots+\sum_{k=1}^{n_{s}}\left[\omega_{k 1}^{(s)}\left|\omega_{k 2}^{(s)}\right| \ldots \mid \omega_{k s}^{(s)}\right]\right) \in R .
$$

We consider such integrals to be functions on $\mathbb{C} \pi_{1}(X, x)$. As such they are not uniquely determined, since two iterated integrals $I$ and $I^{\prime}$ can give the same value on all loops based at $x$. This is the case, if and only if their difference $I-I^{\prime}$ lies in the $\mathbb{C}$-subvector space generated by the following three types of integrals (see e.g. [3], Proposition (1.3)):

(a) $\int d f \omega_{1} \cdots \omega_{r}-\int\left(f \omega_{1}\right) \omega_{2} \cdots \omega_{r}+f(x) \int \omega_{1} \cdots \omega_{r}$,

(b) $\int \omega_{1} \cdots \omega_{i-1} d f \omega_{i} \cdots \omega_{r}-\int \omega_{1} \cdots \omega_{i-1}\left(f \omega_{i}\right) \omega_{i+1} \cdots \omega_{r}$

$$
+\int \omega_{1} \cdots\left(f \omega_{i-1}\right) \omega_{i} \cdots \omega_{r},
$$

(c) $\int \omega_{1} \cdots \omega_{r} d f-f(x) \int \omega_{1} \cdots \omega_{r}+\int \omega_{1} \cdots \omega_{r-1}\left(f \omega_{r}\right)$

with $\omega_{1}, \ldots, \omega_{r} \in \Gamma\left(X, \mathcal{A}_{X}^{1}\right)$ and $f \in \Gamma\left(X, \mathcal{A}_{X}^{0}\right)$.

This is equivalent to saying that the involved 1-forms define an element of $R$.

There is a mixed Hodge structure on $\mathrm{H}^{0}\left(B_{s}\left(\mathcal{A}^{\bullet}\right)\right)$ given by the following filtrations (for details see [3]): With $\mathrm{F}^{p} B\left(\mathcal{A}^{\bullet}\right)$ denoting the set of $\mathbb{C}$-linear combinations of iterated integrals, such that each summand contains at least $p d z^{\prime} s$, we define

$$
\begin{aligned}
\mathrm{F}^{p} \mathrm{H}^{0}\left(B_{s}\left(\mathcal{A}^{\bullet}\right)\right) & :=B_{s} \mathrm{H}^{0}\left(\mathrm{~F}^{p} B\left(\mathcal{A}^{\bullet}\right)\right), \\
\mathrm{W}_{l} \mathrm{H}^{0}\left(B_{s}\left(\mathcal{A}^{\bullet}\right)\right) & :=\mathrm{H}^{0}\left(B_{l}(X)\right),
\end{aligned}
$$

i.e., the elements of $\mathrm{F}^{p} \mathrm{H}^{0}\left(B_{s}\left(\mathcal{A}^{\bullet}\right)\right)$ (or $\overline{\mathrm{F}}^{p} \mathrm{H}^{0}\left(B_{s}\left(\mathcal{A}^{\bullet}\right)\right)$ ) are those which are represented by an iterated integral in $\mathrm{F}^{p} B\left(\mathcal{A}^{\bullet}\right)$ (or in $\overline{\mathrm{F}}^{p} B\left(\mathcal{A}^{\bullet}\right)$ ). The elements of $\mathrm{W}_{l} \mathrm{H}^{0}\left(B_{s}\left(\mathcal{A}^{\bullet}\right)\right)$ are those having a representative of length at most $l$, which means that every summand of the representating iterated integral involves at most $l 1$ forms.

Theorem 4.1 (Chen). There is an isomorphism

$$
\mathrm{H}^{0}\left(B_{s}\left(\mathcal{A}^{\bullet}\right)\right) \longrightarrow \operatorname{Hom}\left(\frac{\mathbb{C} \pi_{1}(X, x)}{J^{s+1}}, \mathbb{C}\right),
$$

given by integrating (classes of) iterated integrals over elements of $\mathbb{C} \pi_{1}(X, x)$.

This is a well-defined map, since the integration of an iterated integral of length at most $s$ over an element of $J^{s+1}$ gives zero.

This isomorphism induces a dual mixed Hodge structure on $\mathbb{C} \pi_{1}(X, x) / J^{s+1}$, via

$$
\mathrm{F}^{p} \operatorname{Hom}_{\mathbb{C}}=\left\{\Phi \mid \Phi\left(\mathrm{F}^{1-p}\left(\mathbb{C} \pi_{1}(X, x) / J^{s+1}\right)\right)=0\right\}
$$

and

$$
\mathrm{W}_{l} \operatorname{Hom}_{\mathbb{Q}}=\left\{\Phi \mid \Phi\left(\mathrm{W}_{-1-l}\left(\mathbb{C} \pi_{1}(X, x) / J^{s+1}\right)\right)=0\right\} .
$$




\section{Illustration: The $J^{2}$-CASE}

Let $(V, \nabla)$ be a flat bundle of rank $r$ with monodromy representation $\rho$, whose $\mathbb{C}$-linear extension, again denoted by $\rho$, factors through $J^{2}$. There is an isomorphism class of Higgs bundles corresponding to $(V, \nabla)$ via Simpson's correspondence Lemma 2.4

Since $\rho$ factors through $J^{2}$, it follows that $(V, \nabla)$ is an extension of some $\left(\mathcal{A}_{X}^{0}, d\right)^{\oplus r_{1}}$ by $\left(\mathcal{A}_{X}^{0}, d\right)^{\oplus r_{2}}$ with $r_{1}+r_{2}=r$.

By Remark 2.5, $(V, \nabla)$ is of the form $(V, D+\eta)$, where $(V, D)$ is a harmonic bundle (hence semi-simple) and $\eta$ is in $\Gamma\left(X, \mathcal{A}_{X}^{1}(\operatorname{End}(V))\right)$, satisfying

$$
D^{\prime} \eta=0 \text { and } D^{\prime \prime} \eta+\eta \wedge \eta=0 .
$$

Since $(V, D)$ is unipotent, it must hold that $(V, D) \cong\left(\mathcal{A}_{X}^{0}, d\right)^{\oplus r}$.

Hence there is a global flat $\mathcal{C}^{\infty}$-frame $f=\left(f_{1}, \ldots, f_{r}\right)$ for $(V, D)$, with respect to which $D=d$, hence $D^{\prime}=\partial$ and $D^{\prime \prime}=\bar{\partial}$, applied coordinatewise.

With respect to the same frame the connection matrix $A$ of $\nabla=d+A$ has the form

$$
A=\left(\begin{array}{cc}
0 & \omega \\
0 & 0
\end{array}\right)
$$

where $\omega=\left(\omega_{i j}\right)$ is a $r_{1} \times r_{2}$-matrix of 1 -forms. The integrability condition implies that all 1-forms $\omega_{i j}$ are closed.

The operator $\eta=\nabla-D$ must be given by the connection matrix $A$.

The operators $D, D^{\prime}$, and $D^{\prime \prime}$ operating on $\eta$ are the induced ones on $\operatorname{End}(V)$. Therefore, the application of the operators $D^{\prime}$ and $D^{\prime \prime}$ on $\eta$ is also the coordinatewise application of $\partial$ and $\bar{\partial}$, and the condition (5.1) implies that $\partial \omega_{i j}=0$ and $\bar{\partial} \omega_{i j}=0$, i.e., the $\omega_{i j}$ are harmonic 1 -forms.

Now $\left(V, D, D^{\prime}, D^{\prime \prime}, \eta\right)$ is the bundle in the category $\hat{\mathcal{C}}_{\text {harm }}^{s}$, defined in Example 2.1, corresponding to $(V, \nabla)$.

The corresponding Higgs bundle via Simpson's correspondence Lemma 2.4 is

$$
\left(V, D^{\prime \prime}+\eta\right)=\left(\bigoplus_{i=1}^{r} f_{i} \mathcal{A}_{X}^{0}, \bar{\partial}+\left(\begin{array}{cc}
0 & \omega \\
0 & 0
\end{array}\right)\right)
$$

The following lemma is a special case of Lemma 6.1

Lemma 5.1. (1) The underlying holomorphic bundle of $\left(V, D^{\prime \prime}+\eta\right)$ is trivial if and only if $\eta$ is of type $(1,0)$, i.e., if and only if all the forms $\omega=\left(\omega_{i j}\right)$ in the formula (5.2) are of type $(1,0)$.

(2) The Higgs field of $\left(V, D^{\prime \prime}+\eta\right)$ is equal to zero if and only if $\eta$ is of type $(0,1)$, i.e., if and only if all the forms $\omega=\left(\omega_{i j}\right)$ in the formula (5.2) are of type $(0,1)$.

With the notation above, the monodromy representation induces the well-defined homomorphism

$$
\begin{aligned}
\bar{\rho}: \frac{\mathbb{C} \pi_{1}(X, x)}{J^{2}} & \longrightarrow \mathrm{M}(r, \mathbb{C}) \\
{[c] } & \longrightarrow \mathrm{E}_{r}+\left(\begin{array}{cc}
0 & \int_{c} \omega_{i j} \\
0 & 0
\end{array}\right) .
\end{aligned}
$$


Since in this case the Hodge filtration on $\mathbb{C} \pi_{1}(X, x) / J^{2}$ is

$$
\mathrm{F}^{i}\left(\mathbb{C} \pi_{1}(X, x) / J^{2}\right)= \begin{cases}0, & i=1, \\ \mathbb{C} \oplus\left\{c \in J / J^{2} \mid \int_{c} \omega=0 \forall \text { hol. } \omega\right\}, & i=0, \\ \mathbb{C} \pi_{1}(X, x) / J^{2}, & i=-1,\end{cases}
$$

and the ideals $I$ and $\bar{I}$ as defined in the introduction are

$$
I=\left\{c \in J / J^{2} \mid \int_{c} \omega=0 \text { for all holomorphic } \omega\right\},
$$

and

$$
\bar{I}=\left\{c \in J / J^{2} \mid \int_{c} \omega=0 \text { for all anti-holomorphic } \omega\right\},
$$

the if-parts of Theorems 1.1 and 1.2 are easily implied by Lemma 5.1

Conversely, the factorization through $I$ (or $\bar{I}$ ) implies that $\int \omega_{i j} \in \mathrm{F}^{1} \mathrm{H}^{0}$ (or in $\overline{\mathrm{F}}^{1} \mathrm{H}^{0}$ ). By the duality of the mixed Hodge structures we first know that there are $\mathcal{C}^{\infty}$-functions $\psi_{i j}$ such that $\omega_{i j}-d \psi_{i j}$ are holomorphic (or anti-holomorphic), since there must be a representative of $\int \omega_{i j}$ in $\mathrm{H}^{0}\left(B_{1}\left(\mathcal{A}^{\bullet}\right)\right)$ with this property. As the $\omega_{i j}$ are harmonic, it follows from the Hodge decomposition theorem that $d \psi_{i j}=0$, such that Lemma 5.1 implies the assertion.

\section{The General CASE}

Let $(V, \nabla)$ be a flat bundle of rank $r$ with monodromy representation $\rho$, whose $\mathbb{C}$ linear extension, which we denote again by $\rho$, factors through $J^{s+1}$. By Propositions 3.1 and 3.3. such a bundle is unipotent, and equivalently a successive extension of direct sums $\left(\mathcal{A}_{X}^{0}, d\right)^{\oplus r_{i}}, i=1, \ldots, s+1$, of trivial flat bundles with $r=\sum_{i=1}^{s+1} r_{i}$.

By Remark 2.5, we know that $(V, \nabla)$ is of the form $(V, D+\eta)$, where $\left(V, D, D^{\prime}, D^{\prime \prime}\right)$ is a harmonic bundle and $\eta \in \Gamma\left(X, \mathcal{A}_{X}^{1}(\operatorname{End}(V))\right)$, satisfying

$$
D^{\prime} \eta=0 \text { and } D^{\prime \prime} \eta+\eta \wedge \eta=0 .
$$

By [7, Theorem $1,(V, D)$ is semisimple. Since it is in addition unipotent, it must hold that $(V, D) \cong\left(\mathcal{A}_{X}^{0}, d\right)^{\oplus r}$.

Therefore there is a global $D$-flat frame $f=\left(f_{1}, \ldots, f_{r}\right)$ for $V$, and with respect to this frame $D=d$, hence $D^{\prime}=\partial$ and $D^{\prime \prime}=\bar{\partial}$, applied coordinatewise.

With respect to the same frame, $\nabla$ is of the form $d+A$ with a connection matrix

$$
A=\left(\begin{array}{ccccc}
0 & \omega_{12} & \omega_{13} & \cdots & \omega_{1, s+1} \\
0 & 0 & \omega_{23} & \cdots & \omega_{2, s+1} \\
\vdots & & & & \vdots \\
0 & 0 & 0 & \cdots & \omega_{s, s+1} \\
0 & 0 & 0 & \cdots & 0
\end{array}\right),
$$

where the $\omega_{i j}$ are $r_{i} \times r_{j}$-matrices of 1 -forms, and the operator $\eta=\nabla-D$ is given by the connection matrix $A$.

The conditions (6.1) become

$$
\partial A=0 \text { and } \bar{\partial} A+A \wedge A=0,
$$

where $\partial$ and $\bar{\partial}$ are applied coordinatewise. 
The corresponding Higgs bundle, via Simpson's correspondence Lemma 2.4 is

$$
\left(V, D^{\prime \prime}+\eta\right)=\left(\bigoplus_{i=1}^{r} f_{i} \mathcal{A}_{X}^{0}, \bar{\partial}+A\right) .
$$

Lemma 6.1. (1) The underlying holomorphic bundle of $\left(V, D^{\prime \prime}+\eta\right)$ is trivial if and only if $\eta$ is of type $(1,0)$, i.e., if and only if the matrix $A$ is of type $(1,0)$.

(2) The Higgs field of $\left(V, D^{\prime \prime}+\eta\right)$ is equal to zero if and only if $\eta$ is of type $(0,1)$, i.e., if and only if the matrix $A$ is of type $(0,1)$.

Proof. The assertion (2) is trivial. Furthermore, it is clear that the underlying holomorphic bundle of $\left(V, D^{\prime \prime}\right)$ is trivial, if $\eta$ is of type $(0,1)$. Conversely, we denote the $(0,1)$-part of $\eta$ by $\eta^{0,1}$, and we assume that there exist global sections $h_{1}, \ldots, h_{r} \in \Gamma(X, V)$ satisfying $\left(D^{\prime \prime}+\eta^{0,1}\right)\left(h_{i}\right)=0$ for all $i$ and forming a global frame for $V$. With respect to the frame $f$, fixed above, the $h_{i}=\sum_{l=1}^{r} \tilde{\phi}_{i l} f_{l}$ have $\mathcal{C}^{\infty}$-coordinates $\tilde{\phi}_{i l}$. Writing

$$
\phi_{i j}=\left(\begin{array}{c}
\tilde{\phi}_{i, r_{1}+\ldots+r_{j-1}+1} \\
\vdots \\
\tilde{\phi}_{i, r_{1}+\ldots+r_{j}}
\end{array}\right)
$$

they satisfy the differential equation

$$
\left(\begin{array}{c}
\bar{\partial} \phi_{i 1} \\
\bar{\partial} \phi_{i 2} \\
\vdots \\
\bar{\partial} \phi_{i, s-1} \\
\bar{\partial} \phi_{i, s} \\
\bar{\partial} \phi_{i, s+1}
\end{array}\right)+\left(\begin{array}{c}
\omega_{12}^{0,1} \phi_{i 2}+\omega_{13}^{0,1} \phi_{i 3}+\cdots+\omega_{1, s+1}^{0,1} \phi_{i, s+1} \\
\omega_{23}^{0,1} \phi_{i 3}+\cdots+\omega_{2, s+1}^{0,1} \phi_{i, s+1} \\
\vdots \\
\omega_{s-1, s}^{0,1} \phi_{i, s}+\omega_{s-1, s+1}^{0,1} \phi_{i, s+1} \\
\omega_{s, s+1}^{0,1} \phi_{i, s+1} \\
0
\end{array}\right)=0
$$

where $\omega_{i j}^{0,1}$ are the entries of $A^{0,1}$, if we split $A=A^{1,0}+A^{0,1}$ into the parts of type $(1,0)$ and $(0,1)$.

This implies that $\phi_{i, s+1}$ is a globally defined (vector-valued) holomorphic function and therefore constant for all $i$. Let us assume that we already know that $\phi_{i, j}$ is constant for all $i$ and for all $j=s+1, s, \ldots, j_{0}$. Denote the entry in the $\left(j_{0}-1\right)$-st line by $\tau_{j_{0}-1}^{0,1}:=\omega_{j_{0}-1, j_{0}}^{0,1} \phi_{i, j_{0}}+\omega_{j_{0}-1, j_{0}+1}^{0,1} \phi_{i, j_{0}+1}+\cdots+\omega_{j_{0}-1, s+1}^{0,1} \phi_{i, s+1}$. By (6.3) we know that $\partial\left(\tau_{j_{0}-1}^{0,1}\right)=0$. Together with $\bar{\partial}\left(\tau_{j_{0}-1}^{0,1}\right)=\bar{\partial}^{2}\left(\phi_{i, j_{0}-1}\right)=0$ this implies that $\tau_{j_{0}-1}^{0,1}$ is harmonic and in addition $\bar{\partial}$-exact. By the Hodge decomposition theorem for the $\bar{\partial}$-operator, saying that $\operatorname{Ker}\left(\bar{\partial}: \Gamma\left(X, \mathcal{A}_{X}^{0,1}\right) \rightarrow \Gamma\left(X, \mathcal{A}_{X}^{0,2}\right)\right)=$ $\bar{\partial}\left(\Gamma\left(X, \mathcal{A}_{X}^{0}\right)\right) \oplus \operatorname{Harm}^{0,1}(X)$, we obtain that $\bar{\partial} \phi_{i, j_{0}-1}=\tau_{j_{0}-1}^{0,1}=0$, hence $\phi_{i, j_{0}-1}$ is constant for all $i$.

Therefore we obtain by induction that all $\phi_{i j}$ are constant. Since the $r \times r$-matrix $\left(\tilde{\phi}_{i j}\right)$ is regular, the differential equation (6.5) implies that $A^{0,1}=0$. Therefore $A$, and hence $\eta$, is of type $(1,0)$.

By Chen's formula the monodromy representation of $(V, \nabla)$ is given by

$$
\rho=E_{r}+\int A+\int A A+\cdots+\int A^{s} .
$$


It will suffice to prove the factorization properties of the monodromy representation using the matrix representation of the monodromy that we obtain with respect to the $D$-flat frame $f$ of the bundle which was chosen at the beginning of this section.

Lemma 6.2. Let $A$ be a matrix of 1-forms with a block structure as in (6.2), satisfying $d A=0$ and $A \wedge A=0$. If $\rho:=E_{r}+\int A+\int A^{2}+\cdots+\int A^{s}$ is a homotopy functional, when restricted to loops based at $x$, then every summand $\int A^{l}, l=1, \ldots, s$, has this property also.

Proof. We prove the assertion by induction over $s$. The case $s=1$ is trivial. For $s>1$ we only have to look at the $(1, s+1)$-entry of $\rho$, since the other entries split into homotopy invariant summands by induction hypothesis.

The (matrix-valued) entry of $\rho$ in the $(1, s+1)$-place is $\sum_{t=0}^{s-1} I_{t}$, where $I_{0}:=$ $\int \omega_{1, s+1}$ and

$$
I_{t}:=\sum_{1<i_{1}<i_{2}<\cdots<i_{t}<s+1} \int \omega_{1 i_{1}} \omega_{i_{1} i_{2}} \cdots \omega_{i_{t}, s+1}, \quad t=1, \ldots, s-1 .
$$

We want to show that every $I_{t}$ is homotopy invariant when restricted to loops based at $x$. By Section 4 we have to show that

$$
\left(d_{C}+d_{I}\right)\left(\sum_{1<i_{1}<i_{2}<\cdots<i_{t}<s+1}\left[\omega_{1 i_{1}}\left|\omega_{i_{1} i_{2}}\right| \cdots \mid \omega_{i_{t}, s+1}\right]\right) \in R .
$$

But since all $\omega_{i j}$ are closed and because of $A \wedge A=0$, we even obtain

$$
d_{I}\left(\sum_{1<i_{1}<\cdots<i_{t}<s+1}\left[\omega_{1 i_{1}}|\cdots| \omega_{i_{t}, s+1}\right]\right)=0,
$$

and

$$
d_{C}\left(\sum_{1<i_{1}<\cdots<i_{t}<s+1}\left[\omega_{1 i_{1}}|\cdots| \omega_{i_{t}, s+1}\right]\right)=-\sum_{1<i_{1}<\cdots<i_{t}<s+1} \sum_{k=1}^{t} \Lambda\left(i_{1}, \ldots, i_{t}, k\right)=0,
$$

where

$$
\Lambda\left(i_{1}, \ldots, i_{t}, k\right):=\left[\omega_{1 i_{1}}|\cdots| \omega_{i_{k-2}, i_{k-1}}\left|\omega_{i_{k-1}, i_{k}} \wedge \omega_{i_{k}, i_{k+1}}\right| \omega_{i_{k+1}, i_{k+2}}|\cdots| \omega_{i_{t}, s+1}\right]
$$

with $i_{0}:=1$ and $i_{t+1}:=s+1$.

Recall that we defined the ideals

$$
I:=J \cap \mathrm{F}^{0}+J^{2} \cap \mathrm{F}^{-1}+\cdots+J^{s} \cap \mathrm{F}^{-(s-1)}
$$

and

$$
\bar{I}:=J \cap \overline{\mathrm{F}}^{0}+J^{2} \cap \overline{\mathrm{F}}^{-1}+\cdots+J^{s} \cap \overline{\mathrm{F}}^{-(s-1)}
$$

in the introduction, where $\mathrm{F}^{i}$ denotes the Hodge filtration of $\frac{\mathbb{C} \pi_{1}(X, x)}{J^{s+1}}$ defined in (4.4).

For the proof of the Theorems 1.1 and 1.2 stated in the introduction we will need the following remark.

Remark 6.3. As above let $\rho=E_{r}+\int A+\int A^{2}+\cdots+\int A^{s}$ be the monodromy representation of a flat bundle factoring through $I$ (or $\bar{I}$ ) and let $\eta$ be the operator coming from the harmonic category (see Example 2.1 (5), and Remark 2.5), given 
by the matrix $A$ with respect to the frame chosen above. By (6.3) the matrix $A$ fulfills $\partial A=0$ and $\bar{\partial} A+A \wedge A=0$. Denote by $A^{\prime}$ the matrix belonging to the $\left(r_{1}+\cdots+r_{s-1}\right)$-subbundle and by $A^{\prime \prime}$ the matrix belonging to the $\left(r_{2}+\cdots+r_{s}\right)$ quotient bundle. Obviously these satisfy the same formulas as $A$. Therefore they define the uniquely determined operators on the harmonic bundles belonging to the sub- and quotient bundle in the description given in Remark 2.5. Their monodromy representations $E_{r-r_{s+1}}+\int A^{\prime}+\int\left(A^{\prime}\right)^{2}+\cdots+\int\left(A^{\prime}\right)^{s-1}$ and $E_{r-r_{1}}+\int A^{\prime \prime}+\int\left(A^{\prime \prime}\right)^{2}+$ $\cdots+\int\left(A^{\prime \prime}\right)^{s-1}$ factor through $J^{s}$ and through $I$ (or $\bar{I}$ ).

Proof of Theorem 1.1. With the notation fixed at the beginning of this section we have that

$$
(V, \nabla)=\left(\bigoplus_{i=1}^{r} f_{i} \mathcal{A}_{X}^{0}, d^{\oplus r}+A\right)
$$

with the connection matrix $A$ fulfilling $\partial A=0$ and $\bar{\partial} A+A \wedge A=0$, and $\left(\bigoplus_{i=1}^{r} f_{i} \mathcal{A}_{X}^{0}\right.$, $\bar{\partial} \oplus r+A)$ being the corresponding Higgs bundle.

If its underlying holomorphic bundle is trivial, i.e., by Lemma 6.1 if $A$ is of type (1,0), the condition $\bar{\partial} A+A \wedge A=0$ implies that $\bar{\partial} A=0$, since this summand is of type $(1,1)$, whereas the other one is of type $(2,0)$. Therefore all entries of $A$ are holomorphic 1-forms, hence closed.

The monodromy representation of $(V, \nabla)$ is $\rho=E_{r}+\int A+\int A^{2}+\cdots+\int A^{s}$. It follows from the previous lemma that every summand $\int A^{l}, l=1, \ldots, s$, is homotopy invariant, when restricted to loops based at $x$. Since we have shown that the entries of all matrices $\omega_{i j}$ are holomorphic 1-forms, it is clear by (4.2) that the integrals of length $l$ are in $\mathrm{F}^{l} \mathrm{H}^{0}\left(B_{s+1}\left(\mathcal{A}^{\bullet}\right)\right)$, hence the factorization property follows immediately.

Conversely, we assume that $\rho=E_{r}+\int A+\int A^{2}+\cdots+\int A^{s}$ factors through $I$. By Lemma 6.1 we have to show that $A$ is necessarily of type $(1,0)$. We prove this by induction over $s$. If $s=0$, the representation $\rho$ is trivial and corresponds to the trivial Higgs bundle $\left(\mathcal{A}_{X}^{0}, \bar{\partial}\right) \oplus r$.

We denote by $\eta$ the operator from Remark 2.5 belonging to $(V, \nabla)$ satisfying (6.1). Its matrix $A$ with respect to the chosen frame fulfills $\partial A=0$ and $\bar{\partial} A+A \wedge A=$ 0 . By Remark 6.3 and induction hypothesis we can assume that all entries of $A$ except for those in the $(1, s+1)$-block are of type $(1,0)$. The condition $\bar{\partial} A+A \wedge A=0$ implies that $\bar{\partial} \omega_{1, s+1}+\left(\omega_{12} \wedge \omega_{2, s+1}+\cdots+\omega_{s-1, s} \wedge \omega_{s, s+1}\right)=0$, from which it follows that $\bar{\partial} \omega_{1, s+1}=0$, since the other summands are of type $(2,0)$, whereas $\bar{\partial} \omega_{1, s+1}$ cannot have a $(2,0)$-part. By assumption it also holds that $\partial \omega_{1, s+1}=0$. From this it follows that $\omega_{1, s+1}$ is harmonic.

In particular, $\omega_{1, s+1}$ is closed, hence $\int \omega_{1, s+1}$ is a homotopy functional, when restricted to loops based at $x$, factoring through $I$ and even factoring through $\mathrm{F}^{0}\left(\frac{\mathbb{C} \pi_{1}(X, x)}{J^{s+1}}\right)$, since integration over a constant also yields zero. By the duality of the mixed Hodge structures, $\int \omega_{1, s+1}$ lies in $\mathrm{F}^{1} \mathrm{H}^{0}\left(\mathrm{~B}_{1}\left(\mathcal{A}^{\bullet}\right)\right)$. Hence there is a (matrix-valued) $\mathcal{C}^{\infty}$-function $\psi_{1, s+1}$ such that $\omega_{1, s+1}-d \psi_{1, s+1}$ is holomorphic, hence also harmonic as $\mathrm{H}^{0}\left(X, \Omega_{X}^{1}\right)=\operatorname{Harm}^{1,0}(X)$. By the Hodge decomposition theorem, saying that $\operatorname{Ker}\left(d: \Gamma\left(X, \mathcal{A}_{X}^{1}\right) \rightarrow \Gamma\left(X, \mathcal{A}_{X}^{2}\right)\right)=d\left(\Gamma\left(X, \mathcal{A}_{X}^{0}\right)\right) \oplus \operatorname{Harm}^{1}(X)$, we obtain that $d \psi_{1, s+1}$, being harmonic and $d$-exact at the same time, equals zero. Thus $\omega_{1, s+1}$ is holomorphic, which completes the proof. 
Proof of Theorem 1.2. With the notation above we have that

$$
(V, \nabla)=\left(\bigoplus_{i=1}^{r} f_{i} \mathcal{A}_{X}^{0}, d^{\oplus r}+A\right)
$$

with the connection matrix $A$ fulfilling $\partial A=0$ and $\bar{\partial} A+A \wedge A=0$, and $\left(\bigoplus_{i=1}^{r} f_{i} \mathcal{A}_{X}^{0}\right.$, $\bar{\partial}^{\oplus r}+A$ ) being the corresponding Higgs bundle.

If its Higgs field is equal to zero, i.e., by Lemma 6.1 if $A$ is of type $(0,1)$, the condition $\partial A=0$ implies that all entries of $A$ are anti-holomorphic, hence closed.

The monodromy representation of $(V, \nabla)$ is $\rho=E_{r}+\int A+\int A^{2}+\cdots+\int A^{s}$. Since it is homotopy invariant and since $A$ is closed, it follows from Lemma 6.2 that every $\int A^{l}, l=1, \ldots, s$, is also homotopy invariant, when restricted to loops based at $x$.

Since all 1-forms in the matrix $A$ are anti-holomorphic, it is clear that the integrals of length $l$ contain $l d \bar{z}$ 's, i.e., by definition of the Hodge filtration (4.2) they are in $\overline{\mathrm{F}}^{l} \mathrm{H}^{0}\left(B_{s+1}\left(\mathcal{A}^{\bullet}\right)\right)$, hence the factorization property follows immediately.

Conversely, we assume that $\rho=E_{r}+\int A+\int A^{2}+\cdots+\int A^{s}$ factors through $\bar{I}$. By Lemma 6.1 we have to show that $A$ is necessarily of type $(0,1)$. We prove this by induction over $s$. For $s=0$ the representation $\rho$ is trivial and it corresponds to the trivial Higgs bundle $\left(\mathcal{A}_{X}^{0}, \bar{\partial}\right)^{\oplus r}$, having Higgs field equal to zero.

By Remark 6.3 and by induction hypothesis we can assume that all entries of $A$ except for those in the (matrix-valued) $(1, s+1)$-block are of type $(0,1)$. We have to show that $\omega_{1, s+1}$ is harmonic.

By assumption it holds that $\partial \omega_{1, s+1}=0$. It suffices to show that also $\bar{\partial} \omega_{1, s+1}=$ 0 . We split $\omega_{1, s+1}=\omega_{1, s+1}^{1,0}+\omega_{1, s+1}^{0,1}$ into the $(1,0)$ - and the $(0,1)$-part. From the condition $\bar{\partial} \omega_{1, s+1}^{1,0}+\bar{\partial} \omega_{1, s+1}^{0,1}+\left(\omega_{12} \wedge \omega_{2, s+1}+\cdots+\omega_{1, s} \wedge \omega_{s, s+1}\right)=0$, it follows that $\bar{\partial} \omega_{1, s+1}^{1,0}=0$, since it is the only summand of type $(1,1)$. Further, $\partial \omega_{1, s+1}^{0,1}=0$ implies that $\omega_{1, s+1}^{0,1}$ is antiholomorphic, in particular closed. Therefore $\bar{\partial} \omega_{1, s+1}^{0,1}=$ $d \omega_{1, s+1}^{0,1}-\partial \omega_{1, s+1}^{0,1}=0$.

Thus $\omega_{1, s+1}$ is harmonic, in particular closed, and we have that $\int \omega_{1, s+1}$ is a homotopy functional factoring through $\bar{I}$.

Since integration over a constant also yields zero we even know that $\int_{c} \omega_{1, s+1}=0$ for all $c \in \overline{\mathrm{F}}^{0}\left(\mathbb{C} \pi_{1}(X, x) / J^{2}\right)$, i.e., $\int \omega_{1, s+1} \in \overline{\mathrm{F}}^{1} \mathrm{H}^{0}\left(\mathrm{~B}_{1}\left(\mathcal{A}^{\bullet}\right)\right)$ by the duality of the mixed Hodge structures.

Hence there is a $\mathcal{C}^{\infty}$-function $\psi_{1, s+1}$ such that $\omega_{1, s+1}-d \psi_{1, s+1}$ is anti-holomorphic, i.e., every entry lies in $\operatorname{Harm}^{0,1}(X)$. Since $\omega_{1, s+1}$ is also harmonic, we obtain by the Hodge decomposition theorem, $\operatorname{Ker}\left(d: \Gamma\left(X, \mathcal{A}_{X}^{1}\right) \rightarrow \Gamma\left(X, \mathcal{A}_{X}^{2}\right)\right)=$ $d\left(\Gamma\left(X, \mathcal{A}_{X}^{0}\right)\right) \oplus \operatorname{Harm}^{1}(X)$, that $d \psi_{1, s+1}$, being harmonic and $d$-exact at the time, equals zero. Therefore $\omega_{1, s+1}$ must be anti-holomorphic, in particular of type $(0,1)$, hence the operator $\eta$ is of type $(0,1)$.

By Lemma 6.1 it follows that the Higgs field of the corresponding Higgs bundle $\left(V, D^{\prime \prime}+\eta\right)$ is equal to zero.

\section{ACKNOWLEDGEMENTS}

I thank M. Nori for suggesting the problem and H. Esnault for the useful discussions we had at different stages of this work. 


\section{REFERENCES}

[1] W. Goldman, J. Millson, The deformation theory of representations of fundamental groups of compact Kähler manifolds, Inst. Hautes Études Sci. Publ. Math., 67 (1988), 43-96. MR:90b:32041

[2] R. Hain, On a generalizaton of Hilbert's 21st problem, Ann. Sci. École Norm. Sup. (4), 19 (1986), 609-627. MR89a:14013

[3] R. Hain, The geometry of the mixed Hodge structure on the fundamental group, Proc. Symp. Pure Math., 46 (1987), 247-282. MR89g:14010

[4] R. Hain, The de Rham homotopy theory of complex algebraic varieties I, K-Theory, 1 (1987), 271-324. MR88h:14029

[5] J. Morgan, The algebraic topology of smooth algebraic varieties, Inst. Hautes Études Sci. Publ. Math., 48 (1978), 137-204. MR80e:55020

[6] J.-P. Serre, Lie algebras and Lie groups, Lect. Notes in Math., 1500 (1992), Springer. MR93h:17001

[7] C.T. Simpson, Higgs bundles and local systems, Inst. Hautes Études Sci. Publ. Math., 75 (1992), 5-95. MR 94d:32027

Fachbereich 6 - Mathematik, Universität Essen, 45117 Essen, Germany

E-mail address: silke.lekaus@uni-essen.de 